\title{
SIMULTANEOUS SPECTRA OF A COMPLETE SAMPLE OF SOURCES FROM THE PMN SURVEY
}

Preliminary results

\author{
M.G. MINGALIEV, A.M. BOTASHEV, and V.A. STOLYAROV \\ Special Astrophysical Observatory, Russia
}

\section{Introduction}

The broad spectral coverage of radio sources including high frequency data are desirable for a number of reasons. Over the last decade, researchers have identified a new class of very high-redshift objects that are more closely related to normal galaxies than to quasars. Surveys with radio telescopes have been instrumental in finding these extremely distant galaxies, which are barely visible at optical wavelengths. The selection of candidates with steep radio spectra has proven a particularly effective means for finding galaxies with redshifts greater than $\mathrm{z}=2$. Secondly, they uncover flat spectrum sources which are likely to be compact and useful as radio astrometric standards and as well as candidates for further interferometric observations. Third, they provide information about physics and, in particular, about the high-energy particles responsible for radio emission. And last but not least, simultaneous observations at several wavelengths will exclude variability of sources in interpretation of their spectra. Here we present the preliminary results of an ongoing investigation of a complete sample of radio sources from the PMN Survey.

\section{Selection of Sample and Observations}

The adopted criteria for selection of the sample from the PMN Survey (Griffith et al. 1994) were:

- flux density $\geq 200 \mathrm{mJy}$ at PMN Survey frequency $(4.8 \mathrm{GHz})$

$-00^{h} \leq \mathrm{RA} \leq 24^{h} ;-21^{\circ} \leq$ Decl. $\leq-17^{\circ}$

- galactic latitude $|b|>10^{\circ}$ to exclude the galactic plane.

In all, we have selected 262 objects. 
The observations were made at $2.7 \mathrm{~cm}, 3.9 \mathrm{~cm}, 7.6 \mathrm{~cm}, 13 \mathrm{~cm}$, and $31 \mathrm{~cm}$ during three sets in July, August and December 1995 by transit way mode at the North sector of the RATAN-600 radio telescope. Each source was observed 5-8 times. As flux and coordinate standard we have used the strong radio source 1245-197. The assumed flux densities, Right Ascension and Declination are following: $1.24 \mathrm{Jy}, 1.75 \mathrm{Jy}, 3.0 \mathrm{Jy}, 4.0 \mathrm{Jy}$, and $6.3 \mathrm{Jy}$ at $2.7 \mathrm{~cm}, 3.9 \mathrm{~cm}, 7.6 \mathrm{~cm}, 13 \mathrm{~cm}$, and $31 \mathrm{~cm}$ respectively; $\mathrm{RA}_{1950.0}=12^{h} 45^{m} 45^{\mathrm{s}} .22, \operatorname{Dec}_{1950.0}=-19^{\circ} 42^{\prime} 57^{\prime \prime} \cdot 5$

The accuracy of flux density measurements at $2.7 \mathrm{~cm}$ was mostly concern with signal-to-noise ratio. In general, the accuracy at the best sensitivity wavelength $(7.6 \mathrm{~cm})$ is $2-3 \%$ or better. The accuracy at $31 \mathrm{~cm}$ was reduced by strong interference from traffic, satellites and military radar systems. The accuracy for each source is given in Table.

In this paper we present the first result of radio observations of 174 sources from the above mentioned sample. To complete the radio observation the last set is scheduled on October 1996.

\section{Results}

Due to the good sensitivity and resolving power in RA at $7.6 \mathrm{~cm}$ we have improved the RA of these sources too. For most sources $(\sim 70 \%)$ the accuracy in RA equal or better than 0.1 , and 0.2 for the remainder. This result will help us for further optical identification of these objects. The RA determined at this program were compared with positions from the PMN and Texas (Douglus 1987) Surveys. On Figures 1(a, b) one can see histograms of $\Delta \alpha / \mathrm{s}$ normalized by the quadratic sums of the quoted rms uncertainties $\mathrm{s}$ (the RATAN-600 minus the PMN and the Texas positions respectively).

The main results of our program of observations are available by $\mathrm{ftp}$

- site big1.sao.ru

- figure/data/ratan/mingaliev_spectrafig

- table/data/ratan/mingaliev_spectra_table.

For determining spectra of this sources in addition to our data we have used the flux densities from the Texas 0.365 GHz Sky Survey (Douglus 1987). A match between sources in our list (after improving the RA during our observations) and Texas Survey found counterparts for $\sim 80 \%$ of them.

\section{Conclusion}

For 174 radio sources from the PMN Tropical Survey: the flux densities at $2.7 \mathrm{~cm}, 3.9 \mathrm{~cm}, 7.6 \mathrm{~cm}, 13 \mathrm{~cm}$, and $31 \mathrm{~cm}$ were measured; simultaneous 


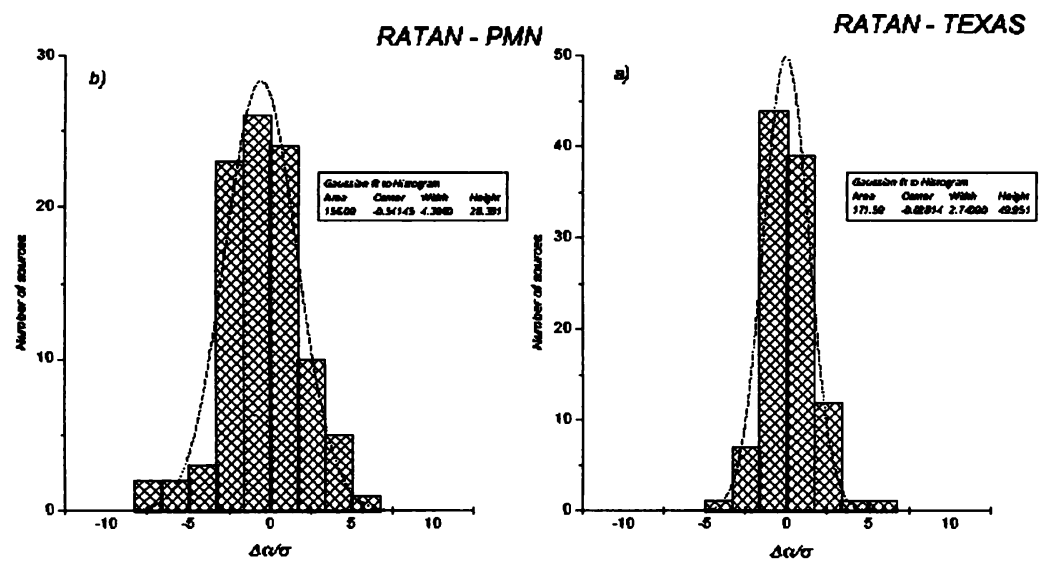

Figure 1.

spectra in wavelength region $2.7-31 \mathrm{~cm}$ were determined; and the accuracy in RA was improved by $5-7$ times.

Further steps are follows: to complete observations of selected sample optical identification with The Digitized Sky Survey (Lasker et al. 1990); identification with other wavebands; to select variable sources comprising the simultaneous spectra obtained by this program with other data from other telescopes observed on different epochs.

\section{Acknowledgements}

This research is partially supported by the Russian Foundation for Basic Research Project No 95-02-04972 and INTAS Project No 94-4010.

\section{References}

Douglus J.N. 1987, Bull.Am.Astron.Soc., 19, 1048

Griffith, M.R., Wright, A.E., Burke, B.F., and Ekers, R.D. 1994, Astrophys.J.Suppl., 90, 179

Lasker, B.M., Sturch, C.R., McLean, B.J., Russell, J.L., Jenkner, H., and Shara M. 1990 Astron.J., 99, 2019 